\title{
A search for ultra-high-energy photons at the Pierre Auger Observatory exploiting air-shower Universality
}

\author{
Pierpaolo Savina ${ }^{a, b, c, d, *}$ on behalf of the Pierre Auger $^{e}$ Collaboration \\ (a complete list of authors can be found at the end of the proceedings) \\ aUniversità del Salento, Dipartimento di Matematica e Fisica “E. De Giorgi”, Lecce, Italy \\ ${ }^{b}$ INFN, Sezione di Lecce, Lecce, Italy \\ ${ }^{c}$ CNRS/IN2P3, IJCLab, Université Paris-Saclay, Orsay, France \\ ${ }^{d}$ University of Wisconsin-Madison, Department of Physics and WIPAC, Madison, WI, USA \\ ${ }^{e}$ Observatorio Pierre Auger, Av. San Martín Norte 304, 5613 Malargüe, Argentina \\ E-mail: spokespersons@auger.org
}

The Pierre Auger Observatory is the most sensitive detector to primary photons with energies above $\sim 0.2 \mathrm{EeV}$. It measures extensive air showers using a hybrid technique that combines a fluorescence detector (FD) with a ground array of particle detectors (SD). The signatures of a photon-induced air shower are a larger atmospheric depth at the shower maximum $\left(X_{\max }\right)$ and a steeper lateral distribution function, along with a lower number of muons with respect to the bulk of hadron-induced background. Using observables measured by the FD and SD, three photon searches in different energy bands are performed. In particular, between threshold energies of 1-10 EeV, a new analysis technique has been developed by combining the FD-based measurement of $X_{\max }$ with the SD signal through a parameter related to its muon content, derived from the universality of the air showers. This technique has led to a better photon/hadron separation and, consequently, to a higher search sensitivity, resulting in a tighter upper limit than before. The outcome of this new analysis is presented here, along with previous results in the energy ranges below $1 \mathrm{EeV}$ and above $10 \mathrm{EeV}$. From the data collected by the Pierre Auger Observatory in about 15 years of operation, the most stringent constraints on the fraction of photons in the cosmic flux are set over almost three decades in energy.

$37^{\text {th }}$ International Cosmic Ray Conference (ICRC 2021)

July 12 th - 23rd, 2021

Online - Berlin, Germany

\footnotetext{
${ }^{*}$ Presenter
} 


\section{Introduction}

Due to magnetic fields that permeate the universe, cosmic rays do not point back to the sources, even at the highest energies. Therefore, the quest for the origin of ultra-high-energy (UHE) cosmic rays intrinsically implies a multi-messenger approach since, on the one hand, direct information about their acceleration sites can be obtained by searching for the neutral particles, $\gamma$-rays and neutrinos, generated by the interactions of cosmic rays at the acceleration sites, via the so-called astrophysical beam dump process [1]. On the other hand, UHE photons are expected to be produced by ultra-high-energy cosmic rays in interaction with the soft photons of the universal backgrounds in their propagation to Earth, via the Greisen-Zatsepin-Kuzmin (GZK) effect for UHE protons and photodisintegration of UHE nuclei [2,3]. These cosmogenic photons also probe ultra-high-energy cosmic rays (CRs) as their flux depends on the characteristics of the sources, as well as on the nature of the parent nuclei. In addition, UHE photons might probe new physics, as their detection would be a smoking gun for, e.g., dark matter composed of super-heavy particles decaying in photons [4].

Due to the steepness of both the cosmic ray and cosmic photon fluxes, this search can presently only be done through large ground-based detectors that exploit the phenomenon of extensive air showers. The identification of photon primaries, when detectors of this kind are used, relies on the ability to distinguish the showers generated by photons from those initiated by the overwhelming background of protons and heavier nuclei. Since the radiation length in the atmosphere is more than two orders of magnitude smaller than the mean free path for photo-nuclear interaction, in photon showers the transfer of energy to the hadron/muon channel is reduced with respect to the bulk of hadron-induced air showers, resulting in a lower number of secondary muons. Additionally, as the development of photon showers is delayed by the typically small multiplicity of electromagnetic interactions, they reach the maximum development of the shower, $X_{\max }$, deeper in the atmosphere with respect to showers initiated by hadrons.

These two observables can be measured at the Pierre Auger Observatory [5], the world's largest cosmic-rays detector, which employs a hybrid detecting technique for the observation of extensive air showers, by combining a fluorescence detector (FD) with a ground array of particle detectors (SD) separated by $1500 \mathrm{~m}$. The FD provides direct observation of the longitudinal shower profile, which allows for the measurement of the energy, $E$, and of the $X_{\max }$ of a shower, while the SD samples the secondary particles at ground level. Although the SD observes showers at a fixed slice in depth, the longitudinal development is embedded in the signals detected. The FD and SD are complemented with the low energy enhancements of the Pierre Auger Observatory, namely the High Elevation Altitude Telescopes (HEAT), i.e., three additional fluorescence telescopes with an elevated field of view, overlooking a denser SD array, in which the stations are separated by $750 \mathrm{~m}$. The combination of these two instruments allow for the measurement of showers in the energy range from below the second knee of the cosmic-ray spectrum up to the ankle.

Three different analyses, differing in the detector used, have been developed to cover the wide energy range inspected by the Pierre Auger Observatory [6, 7]. We present here the overall results of the photon searches developed at the Pierre Auger Observatory, with a focus on a new analysis technique, applied on hybrid events with energies between $1 \mathrm{EeV}$ and $10 \mathrm{EeV}$, which combines the FD based measurement of $X_{\max }$ with a parameter, $F_{\mu}$, related to the muonic content of a shower and derived from the SD station signals by exploiting the air-shower universality. 


\section{Photon searches at the Pierre Auger Observatory}

The photon/hadron separation at the Pierre Auger Observatory is based on parameters sensitive to the muonic content or to the depth of an air shower.

Above $10 \mathrm{EeV}$, the search for UHE photons benefits from the large exposure offered by the SD detector. The analysis is based on the combination of two estimators: the deviation of the measured signals from the average lateral distribution function (LDF) derived from the Auger SD-data and the signal risetime (defined as the time difference between the 50\% and $10 \%$ quantiles of the signal trace). Photon-induced showers present a steeper LDF close to the axis and a larger risetime [6] than showers induced by UHECRs.

Below $10 \mathrm{EeV}$, on average, the number of stations triggered in an event is too small to have a significant separation between photon- and hadron-induced air showers. Nevertheless, due to the increased event statistics, the two low-energy analyses benefit from the Observatory operating in hybrid mode and from the direct measurement of the longitudinal profile of the shower provided by the fluorescence telescopes. Specifically, in the range $10^{17.2} \mathrm{eV}-10^{18.0} \mathrm{eV}$, the analysis relies on the low-energy enhancement of the Observatory: $X_{\max }$ is measured by HEAT, while the number of muons is accessed through a parameter, $S_{b}$, derived from the signals of the SD stations [8]. $S_{b}$ is defined as $S_{b}=\sum_{i} S_{i}\left(\frac{r_{i}}{1000 \mathrm{~m}}\right)^{b}$ where the sum extends over all the triggered stations, $S_{i}$ is the signal measured at the $i$-th station, $r_{i}$ is the distance to the shower axis, and $b$ is a variable exponent. It has been found that the optimal separation between data and photons is obtained by setting $b=4$ [7].

In the range between threshold energies $1-10 \mathrm{EeV}$, instead, we have implemented a new analysis technique that relies on the hybrid measurement in combination with the shower universality. This new approach is discussed in detail in the following section.

\section{Photon-hadron separation through the air-shower Universality}

The general idea behind air-shower universality is that the energy spectrum of the secondary particles produced during the shower development, as well as their angular and lateral distributions, depends mostly on the energy of the primary and on the stage of the shower development [9]. As a consequence, the distribution of secondary particles produced in the cascade can be described at different stages of the shower development so that the distribution of secondary particles at the ground can be predicted.

By exploiting this property, a universality-based model has been developed [10] that allows to predict the signals induced by the secondary particles in the SD stations. This model describes the signal size as the superposition of four components: muons $\left(S_{\mu}\right) ; e^{ \pm}$and $\gamma$ from high energy pions $\left(S_{e \gamma}\right) ; e^{ \pm}$and $\gamma$ from muon decays $\left(S_{e \gamma(\mu)}\right) ; e^{ \pm}$and $\gamma$ due to low energy hadrons $\left(S_{e \gamma(\text { had })}\right)$. Each $i$-th signal component, $S_{\text {comp }}^{i}$, has a universal behavior described as a function of the primary energy $E$, on $X_{\max }$, and on the geometrical configuration of the shower. The relative contributions of each of the four components, $\beta^{i}$, instead, depend on the mass of the primary particle through a parameter representing the number of muons in the shower, $F_{\mu}$. The predicted signal, $S_{\text {pred }}$, can then be expressed as:

$$
S_{\mathrm{pred}}=\sum_{i=1}^{4} \beta^{i}\left(F_{\mu}\right) \cdot S_{\mathrm{comp}}^{i}
$$


where $i$ runs over the four components, while $S_{\text {comp }}^{i}$, in turn, is the contribution of each component and has been parametrised using QGSJetII-03 proton simulations.

Following the approach developed in [11], we exploited the Universality-based signal model in the case of hybrid events. As the hybrid reconstruction provides $E, X_{\max }$, and the shower geometry, $S_{\text {comp }}^{i}$ can be directly calculated for each station involved in a hybrid event. Thus, given the reconstructed signal, $S_{\text {rec}}$, in a station of the SD, $F_{\mu}$ can be calculated for each station in each event by matching $S_{\text {rec }}$ to $S_{\text {pred }}$ in equation (1).

To validate the reconstruction of the $F_{\mu}$ parameter, we used a shower library simulated with CORSIKA [12] using EPOS-LHC [13] as the high-energy hadronic interaction model. Showers initiated by photons and protons are simulated in an energy range from $10^{17.5} \mathrm{eV}$ to $10^{19.5} \mathrm{eV}$ according to a power-law spectrum $E^{-\gamma}$ with $\gamma=1.0$ and zenith angle from $0^{\circ}$ to $65^{\circ}$. Realistic simulations of a hybrid detector with the same configuration of the Pierre Auger Observatory [5] are then performed. The resulting hybrid events are reconstructed following [14]. Selection criteria similar to [7] are applied to ensure a good geometry and longitudinal profile reconstruction. Only showers with reconstructed zenith $\theta<60^{\circ}$ are considered in the following.
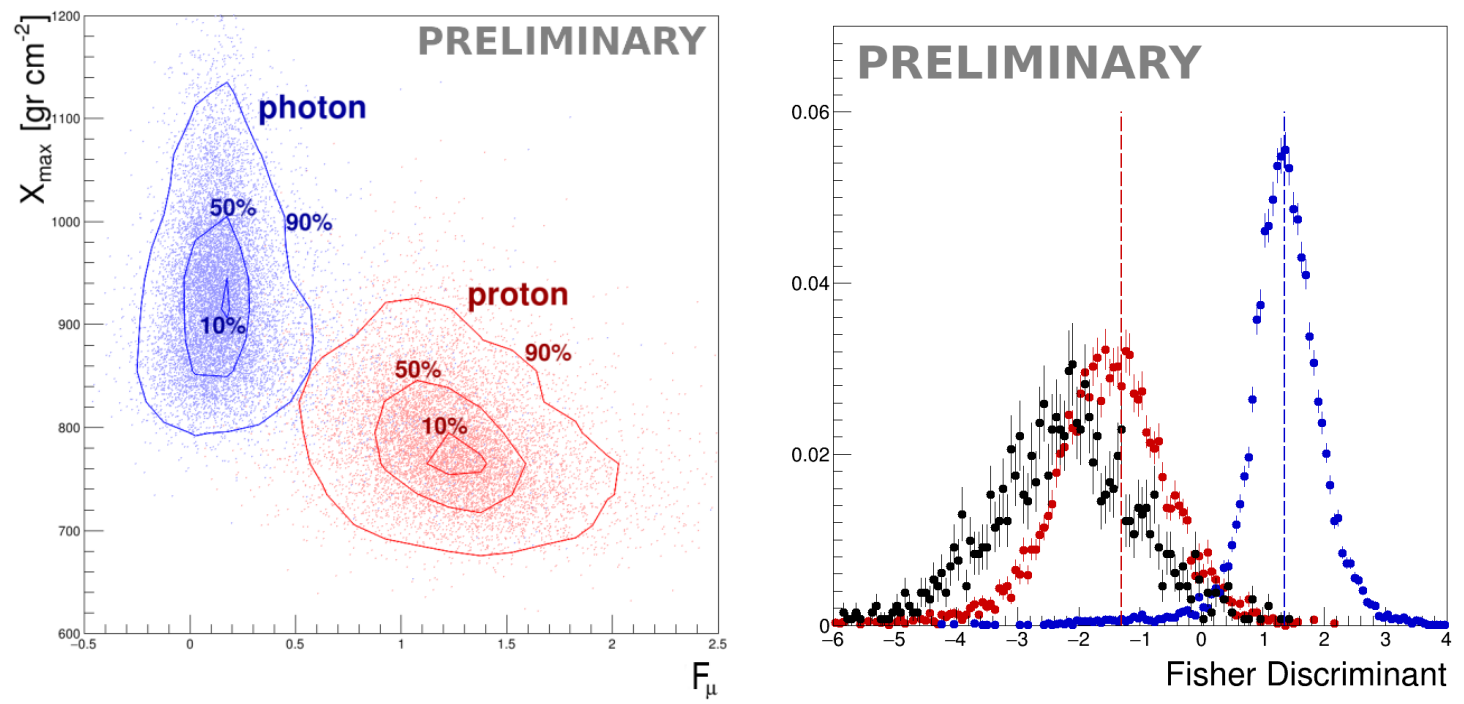

Figure 1: (Left): $X_{\max }-F_{\mu}$ distributions for photons (blue) and protons (red). Contour lines enclose the $90 \%, 50 \%$ and $10 \%$ of the distributions of the events, re-weighted to a realistic power-law spectrum $E^{-\gamma}$ ( $\gamma=2.7$ for protons and $\gamma=2.0$ for photons). (Right): Distribution of the Fisher discriminant for simulated photons (signal, blue) and protons (background, red), and for the burn sample (black). The vertical red line marks the tail of the proton distribution, the blue one indicates the median of the photon distribution.

The median of the $F_{\mu}$ distribution is $\sim 1.3$ for protons and 0.3. Both distributions are characterized by a spread, $\sigma \simeq 0.3$, therefore each median is at more than $3 \sigma$ from the mean value of the other primary type. Since $F_{\mu}$, in hybrid events, provides a very good photon-hadron separation even when derived from the signal of one SD station only, to fully exploit the hybrid approach, we combined it, through a multivariate analysis (MVA) technique, with $X_{\max }$ so to further improve the photon-hadron separation power. The left panel of fig. 1 shows the distributions of $F_{\mu}$ and $X_{\max }$ for photons (blue) and protons (red) in the energy range $10^{18} \mathrm{eV}-10^{19.5} \mathrm{eV}$. The two primaries are well separated with minimal overlap of the tails. $F_{\mu}$ is almost independent of 
the primary energy for both proton and photon primaries. In turn, $X_{\max }$ is linearly increasing with the logarithm of the energy, faster for photons than for protons, implying that its separation power increases with energy.

Thus, we combined $F_{\mu}, X_{\max }$, and energy into a Fisher discriminant analysis [15], that yields the Fisher value, $f$.

The resulting Fisher distributions are shown in the right panel of fig. 1, for protons (red) and photons (blue) primaries. The performance of the discrimination is assessed using simulations. The Fisher discriminant distributions obtained for the proton and photon simulations turned out to be well separated, resulting in a background rejection of $\simeq 99.90 \%$ for a signal efficiency of $50 \%$. Since this analysis has been developed following the prescription of a blind analysis, we exploited a sub-sample of the data, the so-called burn sample (BS), corresponding to 5\% of the full data set (black distribution in the right panel of fig. 1). We firstly compared the Fisher distribution of the burn sample with that of simulated photons, and found them well separated, so that the events contained in the burn sample could be safely considered as background events, and then used to derive a data-driven estimate of the expected background in the full hybrid data sample.

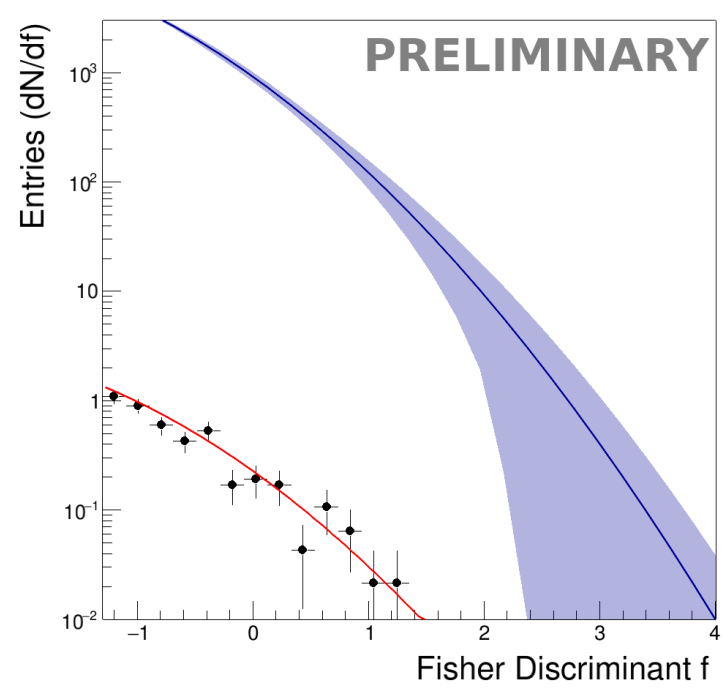

Figure 2: Distribution of the Fisher discriminant for the background expected in the full hybrid data sample (blue curve). The blue shaded area shows the statistical uncertainties on the expected background. For comparison, the corresponding distribution for the burn sample ( $5 \%$ of the full hybrid data set) is also shown (black points), together with the fit to the functional form of the background (red curve).

The background extrapolation procedure is shown in fig. 2: due to the limited number of events in the burn sample, in a preliminary step, we used proton simulations to derive the functional form of the background. By considering only the rightmost tail of the Fisher distribution, specifically only the events with a Fisher discriminant $f_{0}>-1.3$, we found that the background distribution could be described by an exponential with a parabolic exponent (red curve). We then fitted this model to the events of the burn sample (black dots), so to obtain a description of the background, free from either assumptions on cosmic-ray composition or on hadronic interaction models. Then, the extrapolation of the expected background (blue curve) has been obtained by scaling the normalization of the background description to the number of events in the full data sample. The blue shaded area represents the uncertainties on the background description calculated from the propagation of the statistical uncertainties of the fit on the burn sample. The systematic errors on the fit due to a possible photon contamination of the BS have been studied. These uncertainties are negligible with respect to the statistical ones. 
The background estimation was used to derive the photon selection cut on the Fisher discriminant, $f_{\gamma}$. The determination of $f_{\gamma}$ was achieved by studying a proxy for the signal-to-background ratio. The signal was determined by the selection efficiency of photons at different thresholds of the Fisher discriminant, while the background was calculated from the extrapolation of the estimation to the whole data set. The signal to background ratio was found to show a maximum approximately around the median of the Fisher discriminant distribution for photons, that was finally selected as $f_{\gamma}$.

\section{Upper limits on the diffuse photon flux}

The analysis developed has been applied on the hybrid events above $1 \mathrm{EeV}$ collected by the Pierre Auger Observatory between 01 January 2005 and 31 December 2017. Of the total dataset, which consists of approximately 32000 events, the rightmost tail of the Fisher discriminant distribution (black dots) is highlighted in the left panel of fig. 3. The shaded blue bands represent the background extrapolation, including the uncertainty in its estimation for different $\sigma$ levels.

After applying the photon selection cut (dashed vertical line), 22 events are selected. As one can see, the data distribution is consistent with that from the background expectation of $30 \pm 15$ false-positive candidates.
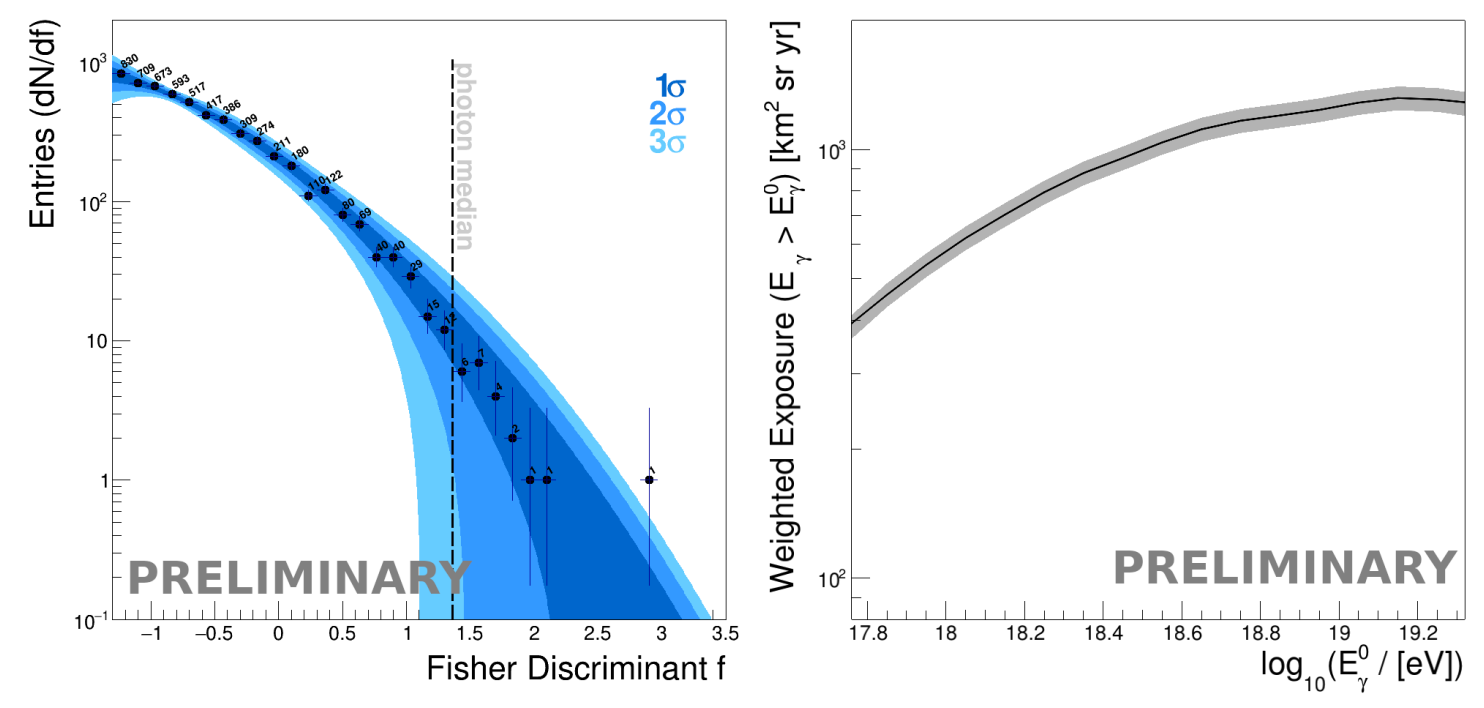

Figure 3: (Left): Tail of the Fisher discriminant distribution of the hybrid data sample (black dots). The vertical dashed line represents the photon-median cut. The shaded blue areas show the 1,2,3 $\sigma$ uncertainties in the expected background. (Right): Weighted hybrid exposure for primary photons (solid line) in the time interval 1 January 2005 - 31 December 2017, assuming a power-law spectrum with $\Gamma=2$. The systematic uncertainty due to the on-time and the trigger efficiency are shown as a gray band.

Since no significant excess of a photon signal with respect to the background was identified, we calculated upper limits to the integral UHE-photon flux, for $E_{\gamma}>E_{\gamma}^{0}$. The upper limits are defined as:

$$
\Phi_{\gamma}^{95 \%}\left(E_{\gamma}>E_{\gamma}^{0}\right)=\frac{N_{\gamma}^{95 \%}}{\langle\mathcal{E}\rangle}
$$




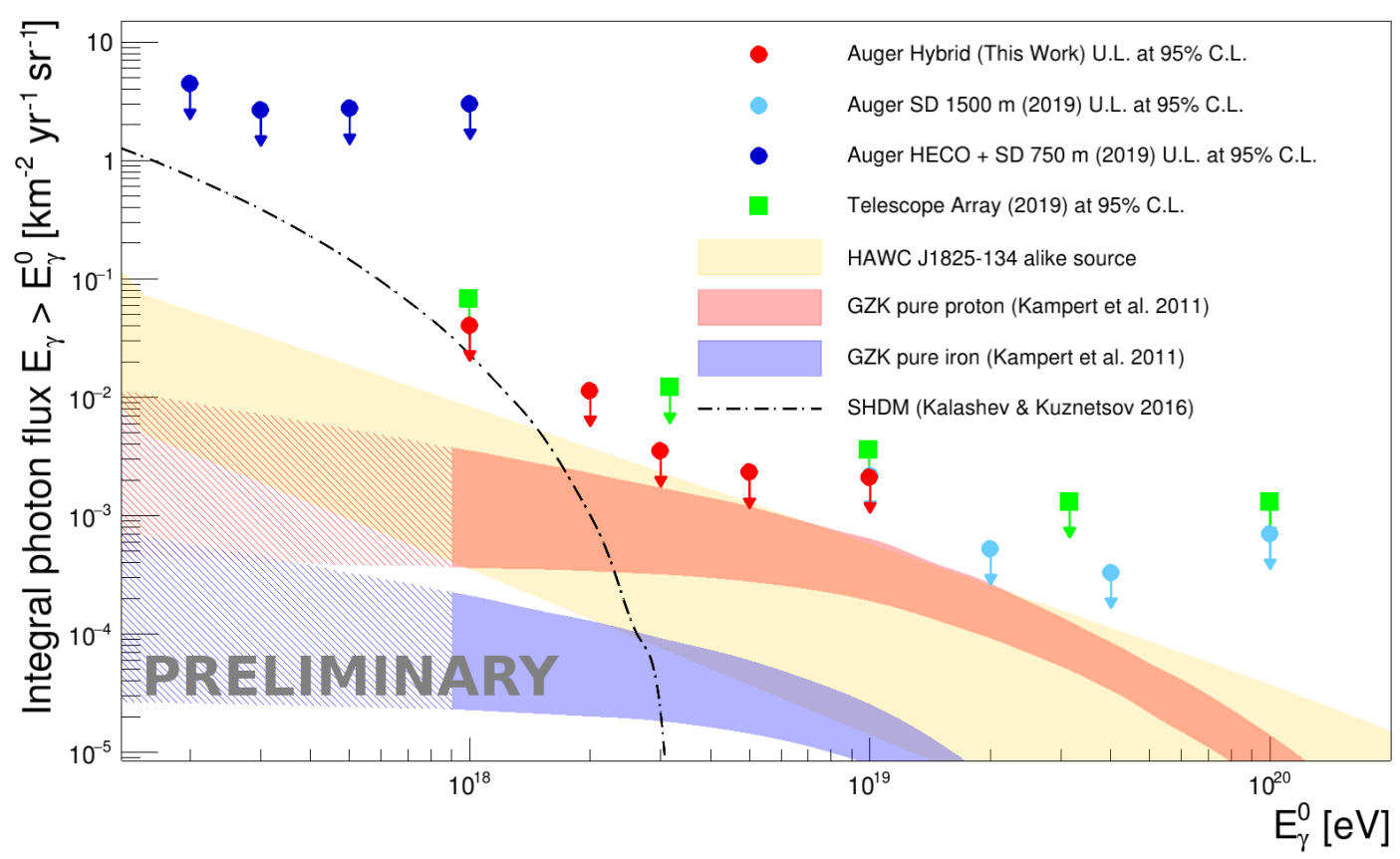

Figure 4: Photon flux limits at $95 \%$ C.L. obtained with this analysis (red circles). Light and dark blue circles show the limits obtained by other Auger analyses using Auger data below $1 \mathrm{EeV}$ and $10 \mathrm{EeV}$ [6]. The light green symbols show the limits derived from Telescope Array data [16]. Predictions of UHE photon fluxes [17] are indicated as colored bands, for comparison. The yellow band shows the extrapolated flux to the highest energies of the source HAWC J1825-134 [18]. The dashed curve shows the flux expected from SHDM particles with mass $M_{\chi}=10^{10} \mathrm{GeV}$ and lifetime $\tau_{\chi}=3 \times 10^{23} \mathrm{yr}$ [4]. More details in the text.

where $E_{\gamma}$ is the reconstructed photon energy, $N_{\gamma}^{95 \%}$ is the Rolke upper limit to the number of photon events computed at $95 \%$ of confidence level, assuming the extrapolated background as background expectation, and $\langle\mathcal{E}\rangle$ is spectrum-weighted average exposure in the energy range $E_{\gamma}>E_{\gamma}^{0}$, shown in the right panel of Fig. 3.

Five different energy thresholds of 1,2,3,5, and $10 \mathrm{EeV}$ were considered. The number of airshower events with photon-like characteristics found at each energy threshold were, respectively, $22,2,0,0,0$. These numbers are compared with the expected background events from a pure hadronic background determined for each energy threshold, respectively: $30 \pm 15,6 \pm 6,0.7 \pm 1.9$, $0.06 \pm 0.25$ and $0.02 \pm 0.06$. Therefore, considering the spectrum-weighted exposure reported in the right panel of fig. 3 , the resulting upper limits in each energy range considered are, respectively, 0.0403, 0.0113, 0.0035, 0.0023, 0.0021 in units of $\mathrm{km}^{-2} \mathrm{sr}^{-1} \mathrm{yr}^{-1}$.

The derived upper limits are the most stringent ones in the energy range from $1 \mathrm{EeV}$ to $10 \mathrm{EeV}$, and are shown in fig. 4, along with previous results below $1 \mathrm{EeV}$ and above $10 \mathrm{EeV}$ obtained in [6].

The attained upper limits can be then discussed in the context of expectations from different GZK expected fluxes for UHE photons, assuming a pure proton (red band) and iron (blue band) composition of the primary cosmic rays. The derived upper limits are found to be approaching the region of the photon fluxes predicted in a pure proton composition scenario. A much larger increase of the exposure would be required to probe scenarios of heavier nuclei.

The yellow band in fig. 4 indicates the extrapolation of the flux observed by the HAWC 
experiment from the source J1825-134 [18], found to be coincident with a giant molecular cloud. HAWC J1825-134 [18] is taken as an exemplary one as its energy spectrum extends well beyond $200 \mathrm{TeV}$ without a clear break or a cutoff. This flux has been calculated by converting it to the flux measured by a diffuse analysis, i.e., ignoring the information related to the arrival direction of the detected events and considering the data period as well as the detector area and the fraction per day where this source is in the field of view of Auger.

A comparison of the achieved upper limits, is also performed with expectations from processes that predict fluxes of photons in non-acceleration (top-down) mechanisms, such as the decay or annihilation of exotic particles, most notably dark matter (DM) composed of super-heavy particles. The latter were recently revived as an alternative to weakly-interacting massive particles. From the absence of photons, we could set constraints on the properties of DM particles in terms of mass $M_{X}$ and lifetime $\tau_{X}$. Nevertheless, even if through our upper limits we can limit the mass and lifetime of SHDM particles, there are still accessible values as shown by the expected flux with the dashed curve, obtained for particles with mass $M_{\chi}=10^{10} \mathrm{eV}$ and lifetime $\tau_{\chi}=3 \times 10^{23} \mathrm{yr}$.

\section{Conclusions}

In this work we discussed a new analysis technique for the search of photon primaries in the $\mathrm{EeV}$ range using hybrid detectors. The attained upper limits are the most stringent ones and are approaching the region of the cosmogenic photon fluxes predicted in a pure proton composition scenario. A larger increase of the exposure would be required instead to probe scenarios of heavier nuclei. The upper limits reported can constrain SHDM models and, in particular, the mass and lifetime of SHDM particles. Nevertheless, these models are not completely excluded.

\section{References}

[1] F. Halzen, Nat. Phys. 13 (2017) 232-238.

[2] K. Greisen, Phys. Rev. Lett. 16 (1966) 748.

[3] G. T. Zatsepin, V. A. Kuz'min, J. Exp. Theor. Phys+ 4 (1966) 78.

[4] O. E. Kalashev, M. Yu. Kuznetsov, Phys. Rev. D. 94 (2016) 063535 [1606. 07354].

[5] A. Aab et al. Nucl. Instrum. Meth. A 798 (2015) 172-213 [1502.01323].

[6] J. Rautemberg et al. [for the Pierre Auger coll.], Proc. 36th Int. Cosmic Rays Conf., PoS(ICRC2019)398.

[7] A. Aab et al. J. Cosmol. Astropart. P. 04 (2017) 009; J. Cosmol. Astropart. P. 09 (2020) E02.

[8] G. Ros et al. Astropart. Phys. 35 (2011) 140-151.

[9] P. Lipari, Phys. Rev. D 79 (2009) 063001.

[10] M. Ave et al. Astropart. Phys. 87 (2017) 23-39.

[11] P. Savina et al. Proc. 36th Int. Cosmic Rays Conf., PoS(ICRC2019)414.

[12] D.Heck et al. Wissenschaftliche Berichte, Forschungszentrum Karlsruhe, FZKA 6019 (1998) 2.

[13] T. Pierog et al. Phys. Rev. C 92 (2015) 034906.

[14] A. Aab et al. J. Cosmol. Astropart. P. 03 (2019) 018;

[15] R. A. Fisher Annals Eugenics, 7 (1936) 7.

[16] R. U. Abbasi et al. Astropart.Phys. 110 (2019) 8-14.

[17] B. Sarkar et al. Proc. 32nd Int. Cosmic Rays Conf., PoS(ICRC2011)198.

[18] A. Albert et al. Astrophys. J. Lett. 907 (2021) L30 [2012 . 15275]. 


\section{The Pierre Auger Collaboration}

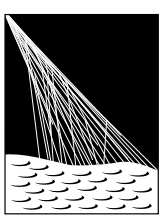

PIERRE

AUSGERVATORY

P. Abreu ${ }^{72}$, M. Aglietta ${ }^{54,52}$, J.M. Albury ${ }^{13}$, I. Allekotte ${ }^{1}$, A. Almela ${ }^{8,12}$, J. Alvarez-Muñiz ${ }^{79}$, R. Alves Batista ${ }^{80}$, G.A. Anastasi ${ }^{63,52}$, L. Anchordoqui ${ }^{87}$, B. Andrada ${ }^{8}$, S. Andringa ${ }^{72}$, C. $\mathrm{Aramo}^{50}$, P.R. Araújo Ferreira ${ }^{42}$, J. C. Arteaga Velázquez ${ }^{67}$, H. Asorey ${ }^{8}$, P. Assis ${ }^{72}$, G. Avila ${ }^{11}$, A.M. Badescu ${ }^{75}$, A. Bakalova ${ }^{32}$, A. Balaceanu ${ }^{73}$, F. Barbato ${ }^{45,46}$, R.J. Barreira Luz $^{72}$, K.H. Becker ${ }^{38}$, J.A. Bellido ${ }^{13,69}$, C. Berat ${ }^{36}$, M.E. Bertaina ${ }^{63,52}$, X. Bertou ${ }^{1}$, P.L. Biermann ${ }^{b}$, V. Binet ${ }^{6}$, K. Bismark ${ }^{39,8}$, T. Bister ${ }^{42}$, J. Biteau ${ }^{37}$, J. Blazek ${ }^{32}$, C. Bleve ${ }^{36}$, M. Boháčová ${ }^{32}$, D. Boncioli ${ }^{57,46}$, C. Bonifazi ${ }^{9,26}$, L. Bonneau Arbeletche ${ }^{21}$, N. Borodai ${ }^{70}$, A.M. Botti ${ }^{8}$, J. Brack ${ }^{d}$, T. Bretz ${ }^{42}$, P.G. Brichetto Orchera ${ }^{8}$, F.L. Briechle ${ }^{42}$, P. Buchholz ${ }^{44}$, A. Bueno ${ }^{78}$, S. Buitink ${ }^{15}$, M. Buscemi ${ }^{47}$, M. Büsken ${ }^{39,8}$, K.S. Caballero-Mora ${ }^{66}$, L. Caccianiga ${ }^{59,49}$, F. Canfora ${ }^{80,81}$, I. Caracas ${ }^{38}$, J.M. Carceller ${ }^{78}$, R. Caruso ${ }^{58,47}$, A. Castellina ${ }^{54,52}$, F. Catalani ${ }^{19}$, G. Cataldi ${ }^{48}$, L. Cazon ${ }^{72}$, M. Cerda ${ }^{10}$, J.A. Chinellato ${ }^{22}$, J. Chudoba ${ }^{32}$, L. Chytka ${ }^{33}$, R.W. Clay ${ }^{13}$, A.C. Cobos Cerutti ${ }^{7}$, R. Colalillo ${ }^{60,50}$, A. Coleman ${ }^{93}$, M.R. Coluccia ${ }^{48}$, R. Conceição ${ }^{72}$, A. Condorelli ${ }^{45,46}$, G. Consolati ${ }^{49,55}$, F. Contreras ${ }^{11}$, F. Convenga ${ }^{56,48}$, D. Correia dos $\operatorname{Santos}^{28}$, C.E. Covault ${ }^{85}$, S. Dasso ${ }^{5,3}$, K. Daumiller ${ }^{41}$, B.R. Dawson ${ }^{13}$, J.A. Day ${ }^{13}$, R.M. de Almeida ${ }^{28}$, J. de Jesús ${ }^{8,41}$, S.J. de Jong ${ }^{80,81}$, G. De Mauro ${ }^{80,81}$, J.R.T. de Mello Neto ${ }^{26,27}$, I. De Mitri ${ }^{45,46}$, J. de Oliveira ${ }^{18}$, D. de Oliveira Franco ${ }^{22}$, F. de Palma ${ }^{56,48}$, V. de Souza ${ }^{20}$, E. De Vito ${ }^{56,48}$, M. del Río ${ }^{11}$, O. Deligny ${ }^{34}$, L. Deval ${ }^{41,8}$, A. di Matteo ${ }^{52}$, C. Dobrigkeit ${ }^{22}$, J.C. D'Olivo ${ }^{68}$, L.M. Domingues Mendes ${ }^{72}$, R.C. dos Anjos ${ }^{25}$, D. dos $\operatorname{Santos}^{28}$, M.T. Dova ${ }^{4}$, J. Ebr ${ }^{32}$, R. Engel ${ }^{39,41}$, I. Epicoco ${ }^{56,48}$, M. Erdmann ${ }^{42}$, C.O. Escobar ${ }^{a}$, A. Etchegoyen ${ }^{8,12}$, H. Falcke ${ }^{80,82,81}$, J. Farmer ${ }^{92}$,

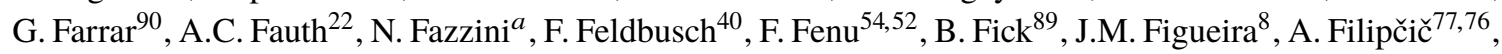
T. Fitoussi ${ }^{41}$, T. Fodran ${ }^{80}$, M.M. Freire ${ }^{6}$, T. Fujii ${ }^{92, e}$, A. Fuster ${ }^{8,12}$, C. Galea ${ }^{80}$, C. Galelli ${ }^{59,49}$, B. García ${ }^{7}$, A.L. Garcia Vegas ${ }^{42}$, H. Gemmeke ${ }^{40}$, F. Gesualdi ${ }^{8,41}$, A. Gherghel-Lascu ${ }^{73}$, P.L. Ghia ${ }^{34}$, U. Giaccari ${ }^{80}$, M. Giammarchi ${ }^{49}$, J. Glombitza ${ }^{42}$, F. Gobbi ${ }^{10}$, F. Gollan ${ }^{8}$, G. Golup ${ }^{1}$, M. Gómez Berisso ${ }^{1}$, P.F. Gómez Vitale $^{11}$, J.P. Gongora ${ }^{11}$, J.M. González ${ }^{1}$, N. González ${ }^{14}$, I. Goos ${ }^{1,41}$, D. Góra ${ }^{70}$, A. Gorgi ${ }^{54,52}$, M. Gottowik ${ }^{38}$, T.D. Grubb $^{13}$, F. Guarino ${ }^{60,50}$, G.P. Guedes ${ }^{23}$, E. Guido ${ }^{52,63}$, S. $\mathrm{Hahn}^{41,8}$, P. Hamal ${ }^{32}$, M.R. Hampel ${ }^{8}$, P. Hansen ${ }^{4}$, D. Harari ${ }^{1}$, V.M. Harvey ${ }^{13}$, A. Haungs ${ }^{41}$, T. Hebbeker ${ }^{42}$, D. Heck ${ }^{41}$, G.C. Hill ${ }^{13}$, C. Hojvat ${ }^{a}$, J.R. Hörandel ${ }^{80,81}$, P. Horvath ${ }^{33}$, M. Hrabovský33 ${ }^{33}$ T. Huege ${ }^{41,15}$, A. Insolia ${ }^{58,47}$, P.G. Isar ${ }^{74}$, P. Janecek ${ }^{32}$, J.A. Johnsen ${ }^{86}$, J. Jurysek ${ }^{32}$, A. Kääpä ${ }^{38}$, K.H. Kampert ${ }^{38}$, N. Karastathis ${ }^{41}$, B. Keilhauer ${ }^{41}$, J. Kemp ${ }^{42}$, A. Khakurdikar ${ }^{80}$, V.V. Kizakke Covilakam ${ }^{8,41}$, H.O. Klages ${ }^{41}$, M. Kleifges ${ }^{40}$, J. Kleinfeller ${ }^{10}$, M. Köpke ${ }^{39}$, N. Kunka ${ }^{40}$, B.L. Lago ${ }^{17}$, R.G. Lang ${ }^{20}$, N. Langner ${ }^{42}$, M.A. Leigui de Oliveira ${ }^{24}$, V. Lenok ${ }^{41}$, A. Letessier-

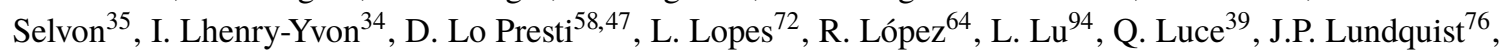
A. Machado Payeras ${ }^{22}$, G. Mancarella ${ }^{56,48}$, D. Mandat ${ }^{32}$, B.C. Manning ${ }^{13}$, J. Manshanden ${ }^{43}$, P. Mantsch ${ }^{a}$, S. Marafico ${ }^{34}$, A.G. Mariazzi ${ }^{4}$, I.C. Mariş ${ }^{14}$, G. Marsella $^{61,47}$, D. Martello ${ }^{56,48}$, S. Martinellii ${ }^{41,8}$, O. Martínez Bravo $^{64}$, M. Mastrodicasa ${ }^{57,46}$, H.J. Mathes ${ }^{41}$, J. Matthews ${ }^{88}$, G. Matthiae ${ }^{62,51}$, E. Mayotte ${ }^{38}$, P.O. Mazur ${ }^{a}$, G. Medina-Tanco ${ }^{68}$, D. Melo ${ }^{8}$, A. Menshikov ${ }^{40}$, K.-D. Merenda ${ }^{86}$, S. Michal ${ }^{33}$, M.I. Micheletti ${ }^{6}$, L. Miramonti ${ }^{59,49}$, S. Mollerach ${ }^{1}$, F. Montanet ${ }^{36}$, C. Morello ${ }^{54,52}$, M. Mostafáa1 ${ }^{91}$, A.L. Müller ${ }^{8}$, M.A. Muller ${ }^{22}$, K. Mulrey ${ }^{15}$, R. Mussa ${ }^{52}$, M. Muzio ${ }^{90}$, W.M. Namasaka ${ }^{38}$, A. Nasr-Esfahani ${ }^{38}$, L. Nellen ${ }^{68}$, M. NiculescuOglinzanu $^{73}$, M. Niechciol ${ }^{44}$, D. Nitz ${ }^{89}$, D. Nosek ${ }^{31}$, V. Novotny ${ }^{31}$, L. Nožka ${ }^{33}$, A Nucita ${ }^{56,48}$, L.A. Núñez ${ }^{30}$, M. Palatka ${ }^{32}$, J. Pallotta ${ }^{2}$, P. Papenbreer ${ }^{38}$, G. Parente ${ }^{79}$, A. Parra ${ }^{64}$, J. Pawlowsky ${ }^{38}$, M. Pech ${ }^{32}$, F. Pedreira ${ }^{79}$, J. Pȩkala ${ }^{70}$, R. Pelayo ${ }^{65}$, J. Peña-Rodriguez ${ }^{30}$, E.E. Pereira Martins ${ }^{39,8}$, J. Perez Armand ${ }^{21}$, C. Pérez

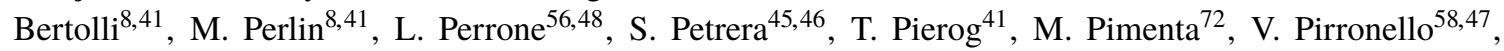
M. Platino ${ }^{8}$, B. Pont $^{80}$, M. Pothast ${ }^{81,80}$, P. Privitera ${ }^{92}$, M. Prouza $^{32}$, A. Puyleart ${ }^{89}$, S. Querchfeld ${ }^{38}$, J. Rautenberg ${ }^{38}$, D. Ravignani ${ }^{8}$, M. Reininghaus ${ }^{41,8}$, J. Ridky ${ }^{32}$, F. Riehn ${ }^{72}$, M. Risse ${ }^{44}$, V. Rizi ${ }^{5746}$, W. Rodrigues de Carvalho ${ }^{21}$, J. Rodriguez Rojo ${ }^{11}$, M.J. Roncoroni ${ }^{8}$, S. Rossoni ${ }^{43}$, M. Roth ${ }^{41}$, E. Roulet ${ }^{1}$, A.C. Rovero ${ }^{5}$, P. Ruehl ${ }^{44}$, A. Saftoiu ${ }^{73}$, F. Salamida ${ }^{57,46}$, H. Salazar ${ }^{64}$, G. Salina ${ }^{51}$, J.D. Sanabria Gomez ${ }^{30}$, F. Sánchez ${ }^{8}$, E.M. Santos ${ }^{21}$, E. Santos ${ }^{32}$, F. Sarazin ${ }^{86}$, R. Sarmento ${ }^{72}$, C. Sarmiento-Cano ${ }^{8}$, R. Sato ${ }^{11}$, P. Savina ${ }^{56,48,34,94}$, C.M. Schäfer ${ }^{41}$, V. Scherini ${ }^{56,48}$, H. Schieler ${ }^{41}$, M. Schimassek ${ }^{39,8}$, M. Schimp ${ }^{38}$, 
F. Schlüter ${ }^{41,8}$, D. Schmidt ${ }^{39}$, O. Scholten ${ }^{84,15}$, P. Schovánek ${ }^{32}$, F.G. Schröder ${ }^{93,41}$, S. Schröder ${ }^{38}$, J. Schulte ${ }^{42}$, S.J. Sciutto ${ }^{4}$, M. Scornavacche ${ }^{8,41}$, A. Segreto ${ }^{53,47}$, S. Sehgal ${ }^{38}$, R.C. Shellard ${ }^{16}$, G. Sigl ${ }^{43}$, G. Silli ${ }^{8,41}$, O. Sima ${ }^{73, f}$, R. Šmída ${ }^{92}$, P. Sommers ${ }^{91}$, J.F. Soriano ${ }^{87}$, J. Souchard ${ }^{36}$, R. Squartini ${ }^{10}$, M. Stadelmaier ${ }^{41,8}$, D. Stanca ${ }^{73}$, S. Stani $i^{76}$, J. Stasielak ${ }^{70}$, P. Stassi ${ }^{36}$, A. Streich ${ }^{39,8}$, M. Suárez-Durán ${ }^{14}$, T. Sudholz ${ }^{13}$, T. Suomijärvi ${ }^{37}$, A.D. Supanitsky ${ }^{8}$, Z. Szadkowski ${ }^{71}$, A. Tapia ${ }^{29}$, C. Taricco ${ }^{63,52}$, C. Timmermans ${ }^{81,80}$, O. Tkachenko ${ }^{41}$, P. Tobiska ${ }^{32}$, C.J. Todero Peixoto ${ }^{19}$, B. Tomé ${ }^{72}$, Z. Torrès ${ }^{36}$, A. Travaini ${ }^{10}$, P. Travnicek ${ }^{32}$, C. Trimarelli ${ }^{57,46}$, M. Tueros ${ }^{4}$, R. Ulrich ${ }^{41}$, M. Unger ${ }^{41}$, L. Vaclavek ${ }^{33}$, M. Vacula ${ }^{33}$, J.F. Valdés Galicia ${ }^{68}$, L. Valore ${ }^{60,50}$, E. Varela ${ }^{64}$, A. Vásquez-Ramírez ${ }^{30}$, D. Veberič ${ }^{41}$, C. Ventura ${ }^{27}$, I.D. Vergara Quispe ${ }^{4}$, V. Verzi ${ }^{51}$, J. Vicha ${ }^{32}$, J. Vink ${ }^{83}$, S. Vorobiov ${ }^{76}$, H. Wahlberg ${ }^{4}$, C. Watanabe ${ }^{26}$, A.A. Watson ${ }^{c}$, M. Weber ${ }^{40}$, A. Weindl ${ }^{41}$, L. Wiencke ${ }^{86}$, H. Wilczyński ${ }^{70}$, M. Wirtz ${ }^{42}$, D. Wittkowski ${ }^{38}$, B. Wundheiler ${ }^{8}$, A. Yushkov $^{32}$, O. Zapparrata ${ }^{14}$, E. Zas ${ }^{79}$, D. Zavrtanik ${ }^{76,77}$, M. Zavrtanik ${ }^{77,76}$, L. Zehrer ${ }^{76}$

${ }^{1}$ Centro Atómico Bariloche and Instituto Balseiro (CNEA-UNCuyo-CONICET), San Carlos de Bariloche, Argentina

${ }^{2}$ Centro de Investigaciones en Láseres y Aplicaciones, CITEDEF and CONICET, Villa Martelli, Argentina

${ }^{3}$ Departamento de Física and Departamento de Ciencias de la Atmósfera y los Océanos, FCEyN, Universidad de Buenos Aires and CONICET, Buenos Aires, Argentina

${ }^{4}$ IFLP, Universidad Nacional de La Plata and CONICET, La Plata, Argentina

${ }^{5}$ Instituto de Astronomía y Física del Espacio (IAFE, CONICET-UBA), Buenos Aires, Argentina

${ }^{6}$ Instituto de Física de Rosario (IFIR) - CONICET/U.N.R. and Facultad de Ciencias Bioquímicas y Farmacéuticas U.N.R., Rosario, Argentina

${ }^{7}$ Instituto de Tecnologías en Detección y Astropartículas (CNEA, CONICET, UNSAM), and Universidad Tecnológica Nacional - Facultad Regional Mendoza (CONICET/CNEA), Mendoza, Argentina

${ }^{8}$ Instituto de Tecnologías en Detección y Astropartículas (CNEA, CONICET, UNSAM), Buenos Aires, Argentina

${ }^{9}$ International Center of Advanced Studies and Instituto de Ciencias Físicas, ECyT-UNSAM and CONICET, Campus Miguelete - San Martín, Buenos Aires, Argentina

${ }^{10}$ Observatorio Pierre Auger, Malargüe, Argentina

11 Observatorio Pierre Auger and Comisión Nacional de Energía Atómica, Malargüe, Argentina

12 Universidad Tecnológica Nacional - Facultad Regional Buenos Aires, Buenos Aires, Argentina

${ }^{13}$ University of Adelaide, Adelaide, S.A., Australia

14 Université Libre de Bruxelles (ULB), Brussels, Belgium

${ }^{15}$ Vrije Universiteit Brussels, Brussels, Belgium

${ }^{16}$ Centro Brasileiro de Pesquisas Fisicas, Rio de Janeiro, RJ, Brazil

${ }^{17}$ Centro Federal de Educação Tecnológica Celso Suckow da Fonseca, Nova Friburgo, Brazil

${ }^{18}$ Instituto Federal de Educação, Ciência e Tecnologia do Rio de Janeiro (IFRJ), Brazil

${ }^{19}$ Universidade de São Paulo, Escola de Engenharia de Lorena, Lorena, SP, Brazil

${ }^{20}$ Universidade de São Paulo, Instituto de Física de São Carlos, São Carlos, SP, Brazil

${ }^{21}$ Universidade de São Paulo, Instituto de Física, São Paulo, SP, Brazil

22 Universidade Estadual de Campinas, IFGW, Campinas, SP, Brazil

${ }^{23}$ Universidade Estadual de Feira de Santana, Feira de Santana, Brazil

${ }^{24}$ Universidade Federal do ABC, Santo André, SP, Brazil

25 Universidade Federal do Paraná, Setor Palotina, Palotina, Brazil

${ }^{26}$ Universidade Federal do Rio de Janeiro, Instituto de Física, Rio de Janeiro, RJ, Brazil

${ }^{27}$ Universidade Federal do Rio de Janeiro (UFRJ), Observatório do Valongo, Rio de Janeiro, RJ, Brazil

28 Universidade Federal Fluminense, EEIMVR, Volta Redonda, RJ, Brazil

${ }^{29}$ Universidad de Medellín, Medellín, Colombia

${ }^{30}$ Universidad Industrial de Santander, Bucaramanga, Colombia

${ }^{31}$ Charles University, Faculty of Mathematics and Physics, Institute of Particle and Nuclear Physics, Prague, Czech Republic

32 Institute of Physics of the Czech Academy of Sciences, Prague, Czech Republic

${ }^{33}$ Palacky University, RCPTM, Olomouc, Czech Republic 
${ }^{34}$ CNRS/IN2P3, IJCLab, Université Paris-Saclay, Orsay, France

${ }^{35}$ Laboratoire de Physique Nucléaire et de Hautes Energies (LPNHE), Sorbonne Université, Université de Paris, CNRSIN2P3, Paris, France

${ }^{36}$ Univ. Grenoble Alpes, CNRS, Grenoble Institute of Engineering Univ. Grenoble Alpes, LPSC-IN2P3, 38000 Grenoble, France

${ }^{37}$ Université Paris-Saclay, CNRS/IN2P3, IJCLab, Orsay, France

38 Bergische Universität Wuppertal, Department of Physics, Wuppertal, Germany

${ }^{39}$ Karlsruhe Institute of Technology (KIT), Institute for Experimental Particle Physics, Karlsruhe, Germany

${ }^{40}$ Karlsruhe Institute of Technology (KIT), Institut für Prozessdatenverarbeitung und Elektronik, Karlsruhe, Germany

${ }^{41}$ Karlsruhe Institute of Technology (KIT), Institute for Astroparticle Physics, Karlsruhe, Germany

${ }^{42}$ RWTH Aachen University, III. Physikalisches Institut A, Aachen, Germany

${ }^{43}$ Universität Hamburg, II. Institut für Theoretische Physik, Hamburg, Germany

44 Universität Siegen, Department Physik - Experimentelle Teilchenphysik, Siegen, Germany

${ }^{45}$ Gran Sasso Science Institute, L’Aquila, Italy

${ }^{46}$ INFN Laboratori Nazionali del Gran Sasso, Assergi (L’Aquila), Italy

${ }^{47}$ INFN, Sezione di Catania, Catania, Italy

${ }^{48}$ INFN, Sezione di Lecce, Lecce, Italy

${ }^{49}$ INFN, Sezione di Milano, Milano, Italy

${ }^{50}$ INFN, Sezione di Napoli, Napoli, Italy

51 INFN, Sezione di Roma "Tor Vergata”, Roma, Italy

52 INFN, Sezione di Torino, Torino, Italy

53 Istituto di Astrofisica Spaziale e Fisica Cosmica di Palermo (INAF), Palermo, Italy

54 Osservatorio Astrofisico di Torino (INAF), Torino, Italy

55 Politecnico di Milano, Dipartimento di Scienze e Tecnologie Aerospaziali , Milano, Italy

56 Università del Salento, Dipartimento di Matematica e Fisica “E. De Giorgi”, Lecce, Italy

${ }^{57}$ Università dell'Aquila, Dipartimento di Scienze Fisiche e Chimiche, L’Aquila, Italy

58 Università di Catania, Dipartimento di Fisica e Astronomia, Catania, Italy

${ }^{59}$ Università di Milano, Dipartimento di Fisica, Milano, Italy

${ }^{60}$ Università di Napoli "Federico II", Dipartimento di Fisica "Ettore Pancini”, Napoli, Italy

${ }^{61}$ Università di Palermo, Dipartimento di Fisica e Chimica "E. Segrè”, Palermo, Italy

62 Università di Roma "Tor Vergata", Dipartimento di Fisica, Roma, Italy

${ }^{63}$ Università Torino, Dipartimento di Fisica, Torino, Italy

${ }^{64}$ Benemérita Universidad Autónoma de Puebla, Puebla, México

${ }^{65}$ Unidad Profesional Interdisciplinaria en Ingeniería y Tecnologías Avanzadas del Instituto Politécnico Nacional (UPIITA-IPN), México, D.F., México

66 Universidad Autónoma de Chiapas, Tuxtla Gutiérrez, Chiapas, México

${ }^{67}$ Universidad Michoacana de San Nicolás de Hidalgo, Morelia, Michoacán, México

${ }^{68}$ Universidad Nacional Autónoma de México, México, D.F., México

${ }^{69}$ Universidad Nacional de San Agustin de Arequipa, Facultad de Ciencias Naturales y Formales, Arequipa, Peru

${ }^{70}$ Institute of Nuclear Physics PAN, Krakow, Poland

${ }^{71}$ University of Łódź, Faculty of High-Energy Astrophysics,Lódź, Poland

${ }^{72}$ Laboratório de Instrumentação e Física Experimental de Partículas - LIP and Instituto Superior Técnico - IST, Universidade de Lisboa - UL, Lisboa, Portugal

73 "Horia Hulubei” National Institute for Physics and Nuclear Engineering, Bucharest-Magurele, Romania

${ }^{74}$ Institute of Space Science, Bucharest-Magurele, Romania

75 University Politehnica of Bucharest, Bucharest, Romania

${ }^{76}$ Center for Astrophysics and Cosmology (CAC), University of Nova Gorica, Nova Gorica, Slovenia

${ }^{77}$ Experimental Particle Physics Department, J. Stefan Institute, Ljubljana, Slovenia

${ }^{78}$ Universidad de Granada and C.A.F.P.E., Granada, Spain

${ }^{79}$ Instituto Galego de Física de Altas Enerxías (IGFAE), Universidade de Santiago de Compostela, Santiago de Compostela, Spain

${ }^{80}$ IMAPP, Radboud University Nijmegen, Nijmegen, The Netherlands 
${ }^{81}$ Nationaal Instituut voor Kernfysica en Hoge Energie Fysica (NIKHEF), Science Park, Amsterdam, The Netherlands

${ }^{82}$ Stichting Astronomisch Onderzoek in Nederland (ASTRON), Dwingeloo, The Netherlands

${ }^{83}$ Universiteit van Amsterdam, Faculty of Science, Amsterdam, The Netherlands

${ }^{84}$ University of Groningen, Kapteyn Astronomical Institute, Groningen, The Netherlands

${ }^{85}$ Case Western Reserve University, Cleveland, OH, USA

${ }^{86}$ Colorado School of Mines, Golden, CO, USA

${ }^{87}$ Department of Physics and Astronomy, Lehman College, City University of New York, Bronx, NY, USA

${ }^{88}$ Louisiana State University, Baton Rouge, LA, USA

${ }^{89}$ Michigan Technological University, Houghton, MI, USA

${ }^{90}$ New York University, New York, NY, USA

${ }^{91}$ Pennsylvania State University, University Park, PA, USA

92 University of Chicago, Enrico Fermi Institute, Chicago, IL, USA

93 University of Delaware, Department of Physics and Astronomy, Bartol Research Institute, Newark, DE, USA

94 University of Wisconsin-Madison, Department of Physics and WIPAC, Madison, WI, USA

${ }^{a}$ Fermi National Accelerator Laboratory, Fermilab, Batavia, IL, USA

${ }^{b}$ Max-Planck-Institut für Radioastronomie, Bonn, Germany

${ }^{c}$ School of Physics and Astronomy, University of Leeds, Leeds, United Kingdom

${ }^{d}$ Colorado State University, Fort Collins, CO, USA

$e^{e}$ now at Hakubi Center for Advanced Research and Graduate School of Science, Kyoto University, Kyoto, Japan

$f$ also at University of Bucharest, Physics Department, Bucharest, Romania 